hep-th/0312037

December, 2003

\title{
Massive Nonlinear Sigma Models and BPS Domain Walls in Harmonic Superspace
}

\author{
Masato Arai ${ }^{a *}$, Evgeny Ivanov ${ }^{b \dagger}$ and Jiri Niederle ${ }^{a} \ddagger$ \\ a Institute of Physics, AS CR, 182 21, Praha 8, Czech Republic \\ ${ }^{b}$ Bogoliubov Laboratory of Theoretical Physics, JINR, Dubna, \\ 141980 Moscow region, Russia
}

\begin{abstract}
Four-dimensional massive $\mathcal{N}=2$ nonlinear sigma models and BPS wall solutions are studied in the off-shell harmonic superspace approach in which $\mathcal{N}=2$ supersymmetry is manifest. The general nonlinear sigma model can be described by an analytic harmonic potential which is the hyper-Kähler analog of the Kähler potential in $\mathcal{N}=1$ theory. We examine the massive nonlinear sigma model with multi-center four-dimensional target hyper-Kähler metrics and derive the corresponding BPS equation. We study in some detail two particular cases with the Taub-NUT and double Taub-NUT metrics. The latter embodies, as its two separate limits, both Taub-NUT and Eguchi-Hanson metrics. We find that domain wall solutions exist only in the double Taub-NUT case including its Eguchi-Hanson limit.
\end{abstract}

*arai@fzu.cz

†eivanov@thsun1.jinr.ru

${ }^{\ddagger}$ niederle@fzu.cz 


\section{Introduction}

It is well known that topological solutions are of importance in various areas of particle physics. Recently, there was renewed interest in such solutions because of their crucial role in the brane world scenario [1, 2, 3]. In this scenario, our world is assumed to be realized on topological objects like domain walls or brane-junctions. Investigating the quantum fluctuation of the domain wall, it was found that zero modes are localized on the wall [4] and the low energy theory becomes a theory on the wall. In other words, domain wall background gives rise to some kind of the dimensional reduction as an alternative to the standard Kaluza-Klein compactification [5]. Supersymmetry (SUSY) can also be implemented in these models, and it is actually a powerful device for constructing their topological solutions. In SUSY theories, these often appear as the BPS states [6] which spontaneously break half of the original SUSY [7]. Therefore, they are

called $\frac{1}{2}$ BPS ones [8]. Viewing the four-dimensional world as a domain wall, we are led to deal with SUSY theories in five dimensions. The minimal possibility is $\mathcal{N}=1, d=5$ SUSY possessing eight supercharges.

SUSY with eight supercharges is very restrictive. For instance, in theories involving only massless scalar multiplets (hypermultiplets), non-trivial interactions can only arise from nonlinearities in kinetic terms. Prior to studying the genuine five-dimensional theories with hypermultiplets, it is instructive to start with similar SUSY theories in four dimensions, i.e., $\mathcal{N}=2, d=4$ theories. Actually, in $\mathcal{N}=1, d=5$ and $\mathcal{N}=2, d=4$ theories, the hypermultiplets contain the same number of on-shell components, viz., two complex scalars and one Dirac fermion. This analysis of the four-dimensional theory could then be of help in studying the brane world scenarios based on SUSY theories in higher dimensions [9, 10].

With regard to rigid $\mathcal{N}=2$ SUSY the target manifold of the hypermultiplet $d=4$ sigma models must be hyper-Kähler (HK) [11]. In these theories, the scalar potential can be obtained only if the hypermultiplets acquire masses by the Scherk-Schwarz mechanism [12] because of the appearance of central charges in the $\mathcal{N}=2$ Poincaré superalgebra [13]. The form of the potential is specified by the norm of the Killing vector of the target manifold isometry whose generator is identified with the central charge [14] (actually, in Ref. [14], the massive HK model on an arbitrary toric HK manifold was given in the component formalism in four-dimensional 
space-time). In Ref. [15], the parallel domain walls solution for the massive $T^{*} \mathbf{C} P^{N-1} \mathrm{HK}$ sigma model was found and its moduli space was constructed. In the same model, dynamics of the domain wall solutions was also studied [16] and the calculation of a number of zero modes was performed [17], using the index theorem. In Ref. [18], a massive nonlinear sigma model with the $T^{*} \mathbf{C} P^{1}$ target was studied in the $\mathcal{N}=1$ and $\mathcal{N}=2$ superfield approaches and its domain wall solutions were examined. In [19], the massive $T^{*} \mathbf{C} P^{N-1} \mathrm{HK}$ model was extended to a massive nonlinear HK model with the cotangent bundle over complex Grassmann manifold as the target space and to some generalization of the latter. The structure of vacua in these models was examined. The lump and Q-lump solutions in the HK nonlinear sigma models were also considered in [20, 21, 22, 23. All models of this type can be called massive nonlinear HK sigma models.

Some massive nonlinear sigma models were studied in an on-shell approach, i.e., by taking account of physical fields only [14, 15, 20, 21, 22, 23]. On the other hand, more appropriate for such a study is an off-shell formalism. It provides a powerful tool of constructing models with the domain wall and brane-junction solutions [24], as well as a low-energy effective action around the wall [25].

The most natural description of $\mathcal{N}=2, d=4$ SUSY field theories is achieved in the harmonic superspace (HSS) [26]. The HSS approach is the only one to allow superfield formulations of $\mathcal{N}=2$ SUSY theories with all supersymmetries being manifest and off-shell. In the HSS approach, any HK nonlinear sigma model can be described by one analytic function which is the HK analog of Kähler potential. This analytic function (HK potential) embodies self-interactions of hypermultiplets. The massless nonlinear sigma models in the HSS formalism were studied in [27, 28, 29]. In particular, in [29], it was shown that the component action obtained from the general massless nonlinear sigma model in HSS coincides with that given in [11, 30]. Central extensions of the nonlinear sigma model with the Taub-NUT (TN) 31] and Eguchi-Hanson (EH) metrics [32] and corresponding mass and scalar potential terms were examined in Refs. 33, 34. The domain wall solutions in $T^{*} \mathbf{C} P^{1}$ were also studied in the harmonic superspace framework, along with the study in $\mathcal{N}=1$ superfield formalism in [18].

The purpose of the present paper is to investigate $\mathcal{N}=2$ massive nonlinear sigma models in the HSS approach. We limit ourselves to the case of sigma models associated with four- 
dimensional HK multi-center metrics, because everything is drastically simplified in this case. The component action (both its kinetic and potential parts) can be written in terms of the single analytic HK potential. The resulting scalar component potential turns out to coincide with that in [14]. The general form of the BPS equation is derived in the multi-center case. As examples we consider sigma models associated with the TN metric and its generalization, the so called double Taub-NUT (DTN) metric (see e.g. 35]). The latter encompasses both the TN and EH metrics as its two limiting cases. We demonstrate that only in the DTN and EH cases BPS domain wall solutions exist. The condition of the existence of SUSY vacua comes out as some restriction on the analytic $\mathrm{HK}$ potential, similarly to the $\mathcal{N}=1$ case where there arise analogous restrictions on the superpotential and Kähler potential. This criterion might be useful in constructing other $\mathcal{N}=2$ models with domain wall solutions.

The paper is organized as follows. Sec. 2 contains a brief review of the general massive nonlinear sigma models in $\mathcal{N}=1$ theory. Sec. 3 is devoted to the $\mathcal{N}=2$ SUSY massive nonlinear sigma model in the HSS approach. We start in subsection 3.1 by recalling basic features of massless $\mathcal{N}=2$ sigma models. The general massive sigma model with at least one isometry is studied in the subsection 3.2. In the subsection 3.3. we study the massive sigma model with $U(1)$ isometry and multi-center HK target manifolds. We show, in this new HSS setting, that the scalar potential is expressed through the norm of the Killing vector. We also study there two particular cases with $U(1)$ isometry. Sec. 4 is devoted to a criterion which the analytic HK potential should satisfy to admit SUSY vacua and to a BPS equation and its solution. The summary and concluding remarks are contained in Sec. 5. In Appendices A and B, we derive the relation between the Killing vectors in harmonic and ordinary spaces, as well as the scalar potential of the massive sigma model with one isometry. In this paper, we basically follow the notation of Ref. [29]. 1

\footnotetext{
${ }^{1} \operatorname{diag}\left(\eta_{\mu \nu}\right)=(1,-1,-1,-1)$ and $\epsilon^{12}=-\epsilon_{12}=-1$.
} 


\section{General massive nonlinear sigma model in $d=4, \mathcal{N}=1$ theory}

The superfield action of general massive $\mathcal{N}=1, d=4$ SUSY nonlinear sigma model is given by the most general off-shell action of chiral $\mathcal{N}=1$ superfields [36, 11, 37].

We denote the chiral scalar superfields, the Kähler potential for the kinetic term and holomorphic superpotential as $\Phi^{i}, K\left(\Phi^{i}, \Phi^{* i}\right)$ and $\mathcal{W}(\Phi)$, respectively. The chiral superfields $\Phi^{i}$ comprises a complex scalar field $A^{i}(x)$, Weyl fermion $\psi^{i}(x)$ and an auxiliary complex scalar field $F^{i}(x)$ :

$$
\Phi^{i}(y, \theta)=A^{i}(y)+\sqrt{2} \theta \psi^{i}(y)+\theta^{2} F^{i}(y), \quad y^{\mu}=x^{\mu}+i \theta \sigma^{\mu} \bar{\theta} .
$$

The Lagrangian is given by

$$
\begin{aligned}
\mathcal{L}= & \int d^{2} \theta d^{2} \bar{\theta} K\left(\Phi, \Phi^{*}\right)+\left[\int d^{2} \theta \mathcal{W}(\Phi)+\text { h.c. }\right] \\
= & g_{i j^{*}}\left(A, A^{*}\right)\left(\partial_{\mu} A^{* j} \partial^{\mu} A^{i}+F^{* j} F^{i}+i \bar{\psi}^{j} \bar{\sigma}^{\mu} D_{\mu} \psi^{i}\right)+\frac{1}{4} g_{i j^{*}, k l^{*}} \psi^{i} \psi^{k} \bar{\psi}^{j} \bar{\psi}^{l} \\
& -F^{i}\left(\frac{1}{2} g_{i m^{*}} \Gamma_{j^{*} k^{*}}^{m^{*}} \bar{\psi}^{j} \bar{\psi}^{k}-\frac{\partial \mathcal{W}}{\partial A^{i}}\right)-F^{* i}\left(\frac{1}{2} g_{m i^{*}} \Gamma_{j k}^{m} \psi^{j} \psi^{k}-\frac{\partial \mathcal{W}^{*}}{\partial A^{* i}}\right) \\
& -\frac{1}{2} \frac{\partial^{2} \mathcal{W}}{\partial A^{i} \partial A^{j}} \psi^{i} \psi^{j}-\frac{1}{2} \frac{\partial^{2} \mathcal{W}}{\partial A^{* i} \partial A^{* j}} \bar{\psi}^{i} \bar{\psi}^{j},
\end{aligned}
$$

where $g_{i j^{*}}=\partial^{2} K / \partial A^{i} \partial A^{* j}$ is the Kähler metric, and $g_{i k^{*}} g^{j k^{*}}=\delta_{i}{ }^{j}$. Here $D_{\mu}$ is the covariant derivative : $D_{\mu} \psi^{i}=\partial_{\mu} \psi^{i}+\Gamma^{i}{ }_{j k} \partial_{\mu} A^{j} \psi^{k}$, where $\Gamma_{j k}^{i}=g^{i l^{*}} \partial_{j} g_{k l^{*}}$ is the holomorphic part of the Levi-Civita connection. We denote the derivation by $g_{j l^{*}, k^{*}} \equiv \frac{\partial g_{j l^{*}}}{\partial A^{* k}}$. Equations of motion for $F^{i}$ are

$$
F^{i}=\frac{1}{2} \Gamma_{j k}^{i} \psi^{j} \psi^{k}-g^{i j^{*}} \frac{\partial \mathcal{W}^{*}}{\partial A^{* j}} .
$$

Substituting (2.3) into (2.2), we obtain

$$
\begin{aligned}
\mathcal{L}= & g_{i j^{*}}\left(A, A^{*}\right)\left(\partial_{\mu} A^{* j} \partial^{\mu} A^{i}+i \bar{\psi}^{j} \bar{\sigma}^{\mu} D_{\mu} \psi^{i}\right)+\frac{1}{4} R_{i j^{*} k l^{*}} \psi^{i} \psi^{k} \bar{\psi}^{j} \bar{\psi}^{l} \\
& -\frac{1}{2} D_{i} D_{j} \mathcal{W} \psi^{i} \psi^{j}-\frac{1}{2} D_{i^{*}} D_{j^{*}} \mathcal{W}^{*} \bar{\psi}^{i} \bar{\psi}^{j}-V\left(A, A^{*}\right)
\end{aligned}
$$

where $R_{i j^{*} k l^{*}}$ is the curvature tensor defined by $R_{i j^{*} k l^{*}}=g_{i j^{*}, k l^{*}}-g_{m n^{*}} \Gamma_{i k}^{m} \Gamma_{j^{*} l^{*}}^{n^{*}}$ and $D_{i}$ is the covariant derivative in the target space :

$$
D_{i} \mathcal{W}=\frac{\partial}{\partial A^{i}} \mathcal{W}
$$




$$
D_{i} D_{j} \mathcal{W}=\frac{\partial^{2}}{\partial A^{i} \partial A^{j}} \mathcal{W}-\Gamma_{i j}^{k} \frac{\partial}{\partial A^{k}} \mathcal{W}
$$

The scalar potential $V\left(A, A^{*}\right)$ is given by

$$
V\left(A, A^{*}\right)=g^{i j^{*}} \frac{\partial \mathcal{W}}{\partial A^{i}} \frac{\partial \mathcal{W}^{*}}{\partial A^{* j}}=g_{i j^{*}} F^{i} F^{* j} .
$$

From (2.7), it is easy to find out the SUSY vacuum condition. The condition of the SUSY vacuum is the vanishing of the scalar potential

$$
0=V\left(A, A^{*}\right)=g^{i j^{*}} \frac{\partial \mathcal{W}}{\partial A^{i}} \frac{\partial \mathcal{W}^{*}}{\partial A^{* j}}=g_{i j^{*}} F^{i} F^{* j}
$$

To simplify things, let us consider the case of the nonlinear sigma model with only one chiral scalar superfield $\Phi$. We find that there are two cases when the SUSY vacuum exists in such a nonlinear sigma model, namely, if

1. there is a stationary point of the superpotential, such that the Kähler metric is not vanishing at this point

$$
\frac{\partial \mathcal{W}}{\partial A}=0, \quad \text { and } \quad g_{A A^{*}} \neq 0 \quad\left(g^{A A^{*}} \neq \infty\right)
$$

2. there is a singularity of the Kähler metric, such that the derivative of the superpotential is not singular at this point

$$
g_{A A^{*}}=\infty \quad\left(g^{A A^{*}}=0\right), \text { and } \quad \frac{\partial \mathcal{W}}{\partial A} \neq \infty .
$$

Note that the vanishing of $F$ term $\left(F^{i}=0\right)$ is neither necessary nor sufficient for SUSY to be unbroken in the nonlinear sigma model, since the Kähler metric in eq. (2.8) can possess zeros and/or singularities.

Next, let us consider the BPS equation. In the following we examine only the bosonic part since we are now interested in the non-trivial bosonic configuration. The BPS equation can be derived from the energy minimum condition. Assuming that there exists a non-trivial configuration along the spatial $y$ axis, the energy of the general nonlinear sigma model (per unit area) is expressed as

$$
E=\int d x_{2}\left(g_{i j^{*}} \partial_{2} A^{* j} \partial_{2} A^{i}+g^{i j^{*}} \frac{\partial \mathcal{W}}{\partial A^{i}} \frac{\partial \mathcal{W}^{*}}{\partial A^{* j}}\right)
$$




$$
\begin{aligned}
& =\int d x_{2}\left\{g_{i j^{*}}\left(\partial_{2} A^{i}-e^{i \delta} g^{i k^{*}} \frac{\partial \mathcal{W}^{*}}{\partial A^{* k}}\right)\left(\partial_{2} A^{* j}-e^{-i \delta} g^{l j^{*}} \frac{\partial \mathcal{W}}{\partial A^{l}}\right)+2 \partial_{2} \operatorname{Re}\left(e^{-i \delta} \mathcal{W}\right)\right\} \\
& \geq 2 \int d x_{2} \partial_{2} \operatorname{Re}\left(e^{-i \delta} \mathcal{W}\right),
\end{aligned}
$$

where $\delta$ is a phase factor. If we impose the most restrictive bound, a phase of the superpotential can be taken as

$$
\delta=\arg (\mathcal{W}(y=\infty)-\mathcal{W}(y=-\infty))
$$

When the bound is satisfied, the following equation must hold

$$
\partial_{2} A^{i}=e^{i \delta} g^{i j^{*}} \frac{\partial \mathcal{W}^{*}}{\partial A^{* j}}
$$

where both sides are evaluated at classical fields. This equation is called the BPS equation, and we thus find that the BPS equation involves both the Kähler potential and the superpotential. So the existence of non-trivial solutions to the BPS equation is encoded in the structure of these entities.

This formulation is useful when we are interested in massive nonlinear sigma models having domain wall solutions. To possess a domain wall solution, the theory should have at least two discrete vacua. The conditions (2.9) and (2.10) can then be used to set up such a model. For instance, in Ref. [24, the model corresponding to the choice $g_{A A^{*}} \equiv K_{A A^{*}}=1 /\left|1-A^{2}\right|$ was given as a simple example. Once the model is specified, one can easily obtain the domain wall solution using (2.13).

\section{General massive nonlinear sigma model in the harmonic superspace}

First, we study the general massive nonlinear sigma model with at least one triholomorphic (i.e., commuting with supersymmetry) $U(1)$ isometry. The presence of such an isometry is necessary if one wishes to gain the mass (and/or scalar potential) terms. We shall not specify how this isometry is realized. Next we examine the particular case of the four-dimensional target HK space. In this case, requiring the theory to have an $U(1)$ isometry implies that the corresponding 
HK metric falls into the multi-center class [35. As was shown in [35], using some coordinate transformation, this $U(1)$ isometry can always be cast in the form in which it is realized as some phase or purely shift transformation of the coordinates of the HK manifold. For this case we shall demonstrate that the scalar potential is given by the square of the isometry Killing vector, in accord with the result of [14].

\subsection{HK sigma model in HSS: the general massless case}

First, we consider the action of the general massless nonlinear sigma model in the HSS approach.

The HSS action for a general nonlinear $\mathcal{N}=2, d=4$ supersymmetric sigma model which yields in the bosonic sector a sigma model with $4 n$ dimensional HK target space is just the general superfield action of $n$ hypermultiplets. In the HSS formalism, the hypermultiplet is described by an analytic superfield $q_{a}^{+}(a=1, \ldots, n$ is a flavor index of fundamental representation of $S p(n))$ which is a function given on the harmonic analytic $\mathcal{N}=2$ superspace

$$
\left\{\zeta_{A}, u_{i}^{ \pm}\right\} \equiv\left\{x_{A}^{\mu}=x^{\mu}-2 i \theta^{(i} \sigma^{\mu} \bar{\theta}^{j)} u_{(i}^{+} u_{j)}^{-}, \theta^{+}=\theta^{i} u_{i}^{+}, \bar{\theta}^{+}=\bar{\theta}^{i} u_{i}^{+}, u_{i}^{ \pm}\right\}
$$

where the coordinates $u^{+i}, u^{-i}, u^{+i} u_{i}^{-}=1, i=1,2^{2}$ are the $S U(2)_{R} / U(1)$ harmonic variables [26, 29].

Exploiting the target space reparameterization covariance, the general action can be cast in the form [29]

$$
S=\frac{1}{2} \int d \zeta_{A}^{(-4)} d u\left[q_{a}^{+} D^{++} q^{a+}+L^{+4}\left(q_{a}^{+}, u_{i}^{ \pm}\right)\right]
$$

where $d \zeta_{A}^{(-4)} d u=d^{4} x_{A} d^{2} \theta^{+} d^{2} \bar{\theta}^{+} d u$ is the measure of integration over analytic superspace (3.1), $D^{++}$is the harmonic covariant derivative defined as

$$
D^{++}=\partial^{++}-2 i \theta^{+} \sigma^{\mu} \bar{\theta}^{+} \partial_{\mu}, \quad \partial^{++}=u^{+i} \frac{\partial}{\partial u^{-i}}
$$

and $L^{+4}\left(q_{a}^{+}, u_{i}^{ \pm}\right)$is the analytic HK potential. The analytic superfield $q_{a}^{+}$can be expanded as

$$
\begin{aligned}
q_{a}^{+}\left(\zeta_{A}, u_{i}^{ \pm}\right)= & F_{a}^{+}+\sqrt{2} \theta^{+} \psi_{a}+\sqrt{2} \bar{\theta}^{+} \bar{\varphi}_{a}+i \theta^{+} \sigma^{\mu} \bar{\theta}^{+} A_{a \mu}^{-}+\theta^{+} \theta^{+} M_{a}^{-}+\bar{\theta}^{+} \bar{\theta}^{+} N_{a}^{-} \\
& +\sqrt{2} \theta^{+} \theta^{+} \bar{\theta}^{+} \bar{\chi}_{a}^{--}+\sqrt{2} \bar{\theta}^{+} \bar{\theta}^{+} \theta^{+} \xi_{a}^{--}+\theta^{+} \theta^{+} \bar{\theta}^{+} \bar{\theta}^{+} P_{a}^{(-3)}
\end{aligned}
$$

\footnotetext{
${ }^{2}$ In what follows, $a, \ldots, f$ stand for the $S p(n)$ indices and $i, j, \ldots$ for the $S U(2)_{R}$ indices, respectively.
} 
and it satisfies the reality condition

$$
\widetilde{q_{a}^{+}}=\Omega^{a b} q_{b}^{+}
$$

Here $\Omega^{a b}$ is the skew-symmetric constant $S p(n)$ metric, $\Omega^{a b} \Omega_{b c}=\delta_{c}^{a}$, and " $\sim$ " denotes a (pseudo) conjugation which is the product of the complex conjugation (denoted by "-") and the star (pseudo) conjugation [29]. The action of the " $\sim$ " conjugation on $\zeta_{A}$ and $u_{i}^{ \pm}$is defined as

$$
\widetilde{x_{A}^{\mu}}=x_{A}^{\mu}, \widetilde{\theta^{+}}=\bar{\theta}^{+}, \widetilde{\bar{\theta}^{+}}=-\theta^{+}, \widetilde{u_{i}^{ \pm}}=u^{ \pm i}, \widetilde{u^{ \pm i}}=-u_{i}^{ \pm}
$$

In what follows, we shall frequently omit the $S p(n), S U(2)_{R}$ and space-time indices of the arguments in the analytic functions, e.g. write $f=f\left(q_{a}^{+}, u_{i}^{ \pm}\right)=f(q, u)$.

The action (3.2) is assumed to be invariant under the following isometry transformation ${ }^{3}$

$$
\delta q^{a+}=\varepsilon \lambda^{a+}(q, u)
$$

provided that $\lambda^{a+}(q, u)$ satisfies the equations $\left(\partial_{a+}=\partial / \partial q^{a+}\right)$

$$
\begin{aligned}
& \lambda^{a+}=\frac{1}{2} \Omega^{a b} \partial_{b+} \Lambda^{++}, \\
& \partial^{++} \Lambda^{++}-\frac{1}{2} \Omega^{a b} \partial_{a+} L^{+4} \partial_{b+} \Lambda^{++}=0 .
\end{aligned}
$$

In eq. (3.7), $\varepsilon$ is a group parameter. The quantities $\lambda^{a+}$ and $\Lambda^{++}$are referred to as the superfield Killing vector and Killing potential, respectively. In what follows we shall need eqs. (3.7) - (3.9) only in the limit when all fermions are discarded, which amounts to the reduction $q^{+} \rightarrow F^{+}$.

From now on, we neglect all fermionic fields and deal with the bosonic component action. Both fermionic and bosonic components in (3.4) contain infinite sets of auxiliary fields coming from the harmonic expansions. In order to obtain the action in terms of $4 n$ physical bosonic fields only, we should eliminate the relevant auxiliary fields by solving their algebraic (i.e., kinematical) equations of motion. Therefore, as the basic steps towards the final sigma model action we should single out the kinematical part of the equations of motion following from (3.2) (with all fermions being discarded) and solve these equations.

\footnotetext{
${ }^{3}$ In general, (3.2) is not obliged to respect any extra symmetry except for $\mathcal{N}=2$ SUSY.
} 
Substituting the bosonic part of Grassmann expansion (3.4) into the action (3.2), and integrating over Grassmann coordinates, we obtain the bosonic action in the form

$$
\begin{aligned}
S_{b o s}= & \int d^{4} x_{A} d u\left\{\frac{1}{4} A_{\mu}^{a-}\left[\left(D^{++} \delta_{a}^{b}-\frac{1}{2} \partial_{a+} \partial_{c+} \Omega^{c b} L^{+4}\right) A_{b}^{-\mu}-4 \partial^{\mu} F_{a}^{+}\right]\right. \\
& \left.-M^{a-}\left(D^{++} \delta_{a}^{b}-\frac{1}{2} \partial_{a+} \partial_{c+} \Omega^{c b} L^{+4}\right) N_{b}^{-}-P^{a(-3)}\left(D^{++} F_{a}^{+}-\frac{1}{2} \partial_{a+} L^{+4}\right)\right\} .
\end{aligned}
$$

Here $L^{+4}=L^{+4}(F, u) \equiv L^{+4}(q, u)$ at $\theta=0$. Equation of motion for $F_{a}^{+}$is

$$
D^{++} F_{a}^{+}(x, u)-\frac{1}{2} \partial_{a+} L^{+4}(F, u)=0 .
$$

Here $D^{++}$coincides with a partial harmonic derivative $\partial^{++}$which acts on the harmonic arguments of the component fields as well as on the harmonics appearing explicitly. We denote the harmonic derivative in (3.11) by $D^{++}$in order to distinguish it, e.g., from the partial derivative in (3.9) which acts only on the explicit harmonics in $\Lambda^{++}(F, u)$ and not on the harmonic arguments of $F=F(x, u)$. We reserve the notation $\partial^{++}$just for this latter derivative.

The derivative $D^{++}$, when applied to an arbitrary scalar function $G(F, u)$, yields:

$$
D^{++} G=\partial^{++} G+D^{++} F^{a+} \partial_{a+} G=\partial^{++} G+\frac{1}{2} \Omega^{a b} \partial_{b+} L^{+4} \partial_{a+} G,
$$

where we used eq. (3.11). Defining

$$
\mathcal{D}^{++} G_{a}=D^{++} G_{a}-\frac{1}{2} \partial_{a+} \partial_{b+} L^{+4} G^{b}
$$

equations of motion for $A_{a \mu}^{-}, M_{a}^{-}$and $N_{a}^{-}$can be rewritten as

$$
\begin{aligned}
& \mathcal{D}^{++} A_{a \mu}^{-}-2 \partial_{\mu} F_{a}^{+}=0, \\
& \mathcal{D}^{++} M_{a}^{-}=\mathcal{D}^{++} N_{a}^{-}=0 .
\end{aligned}
$$

The remaining equation, which comes from the variation with respect to $F_{a}^{+}$, is dynamical, and we will not use it in the following (it can be reproduced in the end by varying the eventual action with respect to the dynamical fields $f^{a i}(x)$ to be defined below).

After substituting eqs. (3.11), (3.14) and (3.15) back into (3.10), the action is drastically simplified

$$
S_{b o s}=\int d^{4} x_{A} d u\left(-\frac{1}{2} A_{\mu}^{a-} \partial^{\mu} F_{a}^{+}\right)
$$


Note that the harmonic fields $F_{a}^{+}$and $A_{a \mu}^{-}$are still subject to the constraints (3.11) and (3.14) and they include infinite sets of auxiliary fields. Solving eq. (3.11), one can express $F_{a}^{+}\left(x_{A}, u\right)$ as $F_{a}^{+}=F_{a}^{+}\left(f^{a i}, u\right)$ where $f^{a i}(x)$ are the standard HK target space coordinates. We shall refer to

$$
f^{a \pm}=f^{a i}(x) u_{i}^{ \pm}
$$

as the "central basis" HK coordinates and to $F^{a+}$ and $F^{a-}$ related by

$$
D^{++} F^{a-}=F^{a+}
$$

as the "analytic basis" HK coordinates [38, 29. A more detailed explanation of this nomenclature can be found in [38, 29]. Given the solution $F_{a}^{+}=F_{a}^{+}\left(f^{a i}, u\right)$, the field $A_{a \mu}^{-}$can be expressed from (13.14) as $A_{a \mu}^{-}=A_{a \mu}^{-}\left(f^{a i}, u\right)$. Substituting these solutions into the action results in the final sigma model action for $f^{a i}(x)$.

In solving eqs. (3.11), (3.14) and (3.15), it is convenient to make use of the one-to-one correspondence between the HK sigma models and the geometric construction of HK manifold in the harmonic space [38, 29]. In the latter formulation, the standard constraints of the HK geometry are interpreted as the conditions of harmonic analyticity. This allows one to solve defining constraints of the HK geometry in terms of two unconstrained analytic potentials one of which proves to be pure gauge. The remaining potential encodes all the information about the given HK manifold, in the sense that all the relevant geometric objects, i.e., connections, vielbeins and metric, can be expressed in terms of this potential. We call this geometric approach the nonLagrangian one, in contrast to the Lagrangian approach to which we adhere in this paper and in which the metric and other geometric quantities of HK geometry appear in the $\mathcal{N}=2, d=4$ supersymmetric sigma model type action as the result of solving equations of motion for an infinite tower of auxiliary fields contained in $q^{a+}$. As was shown in [38, 29, both approaches are in fact equivalent to each other. In particular, the unconstrained potential in the non-Lagrangian approach corresponds to the analytic HK potential in $\mathcal{N}=2$ nonlinear sigma model. Using the one-to-one correspondence between these two approaches, as well as the differential geometry techniques of Refs. [38, 29], it is easy to check that the solution of eqs. (3.14) and (3.15) is

$$
\begin{aligned}
& A_{\mu}^{a-}=2 E_{b i}^{a-} \partial_{\mu} f^{b i}, \\
& M_{a}^{-}=N_{a}^{-}=0,
\end{aligned}
$$


where $E_{b i}^{a-}$ is one of the two central basis vielbeins $E_{b i}^{a \pm}$ from which the HK metric is constructed. They satisfy the relations [29]

$$
\mathcal{D}^{++} E_{b i}^{a+}=0, \quad E_{b i}^{a+}=-\mathcal{D}^{++} E_{b i}^{a-}=-\partial_{b i} F^{a+},
$$

which can be deduced based upon eq. (3.11) (for instance, the first one is proved by applying $\partial / \partial f^{b i}$ to (3.11)). Substituting (3.19) and (3.20) into (3.16), we obtain

$$
S_{\text {bos }}=\frac{1}{2} \int d^{4} x_{A} g_{a i, b j}\left(x_{A}\right) \partial^{\mu} f^{a i} \partial_{\mu} f^{b j},
$$

where $g_{a i, b j}$ is the HK target space metric defined by

$$
g_{a i, b j}=\Omega_{c d}\left(E_{a i}^{c-} E_{b j}^{d+}-E_{a i}^{c+} E_{b j}^{d-}\right) .
$$

It is easy to show that this metric is $u$ independent, $g_{a i, b j}=g_{a i, b j}\left(x_{A}\right)$. This can be checked utilizing eq. (3.21), keeping in mind that the $S p(n)$ connections drop out altogether due to the contraction of the $S p(n)$ indices.

We briefly sketch the basic steps leading from the action (3.16) to the final action (3.22). Firstly, we use in (3.16) the relation

$$
\partial^{\mu} F^{a+}=\partial^{\mu} f^{c k} \partial_{c k} F^{a+}=-\partial^{\mu} f^{c k} E_{c k}^{a+},
$$

after which the integrand in (3.16) is reduced to

$$
\Omega_{a d} E_{b i}^{a-} E_{c k}^{d+} \partial_{\mu} f^{b i} \partial^{\mu} f^{c k}
$$

Then we take into account that the second piece in (3.23) is reduced to the first one under the $u$-integral: one should use eq. (3.21), take into account that the $S p(n)$ connections drop out due to the contractions of the $S p(n)$ indices, and finally integrate by parts with respect to $D^{++}$. After these manipulations the last expression is reduced, modulo a total harmonic derivative, just to the metric term in (3.22).

To close the discussion of the massless nonlinear sigma model, we collect some useful formulas related to the $U(1)$ isometry (3.7) projected on the bosonic sector. The basic HSS Killing potential equation (3.9) on the surface of the kinematic equation (3.11) amounts to the 'conservation law':

$$
D^{++} \Lambda^{++}(F, u)=0
$$


In the central basis, eq. (3.24) implies

$$
\Lambda^{++}(f, u)=\Lambda^{i k}(f) u_{i}^{+} u_{k}^{+}
$$

Using (3.24) and (3.13), it is also easy to find

$$
\mathcal{D}^{++} \lambda^{a+}(F, u)=0
$$

From eqs. (3.7) - (3.9) and also using (3.25), we find the following central basis form of (3.7)

$$
\delta F^{a+}=\varepsilon \lambda^{a+}(F, u)=\varepsilon \frac{1}{2} \Omega^{a b} \partial_{b+} f^{c k} \partial_{c k} \Lambda^{i j}(f) u_{i}^{+} u_{j}^{+}=\varepsilon \partial_{c k} F^{a+} k^{c k}(f)=-\varepsilon E_{c k}^{a+} k^{c k} .
$$

Here $k^{c k}(f)$ is the ordinary Killing vector,

$$
\delta f^{c k}=\varepsilon k^{c k}(f) .
$$

The identity (3.27) allows one to find the explicit expression for $k^{a i}(f)$ in terms of the central basis Killing potential $\Lambda^{(i j)}(f)$ (see Appendix A).

\subsection{General massive HK sigma model in HSS}

Next we consider the general massive deformation of the HSS $q^{+}$Lagrangian. Suppose we are given a $q^{+}$action possessing an isometry. Then we assign to $q^{+}$a dependence on the central charge coordinate $x^{5}$, such that $\partial / \partial x^{5}$ can be identified with the Killing vector of the isometry

$$
\frac{\partial}{\partial x^{5}} q^{a+}=m \lambda^{a+}(q, u)
$$

where $m$ is a mass parameter which, for simplicity, is taken to be real. Correspondingly, the harmonic covariant derivative (3.3) acquires the central charge term [39]:

$$
D^{++} \quad \rightarrow \quad D^{++}+i\left[\left(\theta^{+}\right)^{2}-\left(\bar{\theta}^{+}\right)^{2}\right] \frac{\partial}{\partial x^{5}}
$$

and the action (3.2) is modified as

$$
S=\frac{1}{2} \int d \zeta_{A}^{(-4)} d u\left(q_{a}^{+} D^{++} q^{a+}+L^{+4}(q, u)+i m\left[\left(\theta^{+}\right)^{2}-\left(\bar{\theta}^{+}\right)^{2}\right] q_{a}^{+} \lambda^{a+}\right) .
$$


Similarly to the massless case, substituting the Grassmann expansion of the harmonic superfield (3.4) with suppressed fermions into (3.31), we obtain the bosonic action in the following form

$$
\begin{aligned}
S_{b o s} & =\int d^{4} x_{A} d u\left(\frac{1}{4} A_{\mu}^{a-}\left(\mathcal{D}_{a}^{++b} A_{b}^{-\mu}-4 \partial^{\mu} F_{a}^{+}\right)-M^{a-} \mathcal{D}_{a}^{++b} N_{b}^{-}\right. \\
& \left.-P^{a(-3)}\left(D^{++} F_{a}^{+}-\frac{1}{2} \partial_{a+} L^{+4}\right)+i m\left(N^{a-} \partial_{a+}\left(F_{b}^{+} \lambda^{b+}\right)-M^{a-} \partial_{a+}\left(F_{b}^{+} \lambda^{b+}\right)\right)\right) .(3 .
\end{aligned}
$$

The corresponding equations of motion read

$$
\begin{aligned}
& \mathcal{D}^{++} M_{a}^{-}-i m \partial_{a+}\left(F_{b}^{+} \lambda^{b+}\right)=0 \\
& \mathcal{D}^{++} N_{a}^{-}+i m \partial_{a+}\left(F_{b}^{+} \lambda^{b+}\right)=0
\end{aligned}
$$

along with (3.11) and (3.14). It is worth emphasizing that the equations of motion for $M_{a}^{-}$and $N_{a}^{-}$are modified as compared to (3.15) due to the mass deformation, while the equations (3.11) and (3.14) for $F_{a}^{+}$and $A_{a \mu}^{-}$are not modified. As in the massless case, these equations serve to express infinite sets of auxiliary fields collected in the involved quantities in terms of the central basis HK coordinate $f^{a i}(x)$ and its $x$-derivative.

After substituting these kinematical equations of motion into (3.32), the bosonic component action acquires the simple form

$$
S_{\text {bos }}=\int d^{4} x_{A} d u\left[-\frac{1}{2} A_{\mu}^{a-} \partial^{\mu} F_{a}^{+}-\frac{i m}{2}\left(M^{a-}-N^{a-}\right) \partial_{a+}\left(F_{b}^{+} \lambda^{b+}\right)\right] .
$$

The harmonic fields in (3.35) are still solutions of (3.11), (3.14), (3.33) and (3.34). Since we have already solved the equations (3.11) and (3.14) while studying the massless case, the remaining equations to be solved are (3.33) and (3.34). Here we present the general form of the solution for $M^{a-}, N^{a-}$ (some details of the derivation are given in Appendix B):

$$
M^{a-}=-N^{a-}=i m\left(\mathcal{F}_{b}^{-} \partial_{+}^{a} \lambda^{b+}+k^{c i}\left[\partial_{c i} \mathcal{F}^{a-}-2 E_{c i}^{a-}\right]\right)
$$

Here $\mathcal{F}_{a}^{-}$is defined by

$$
F_{a}^{+}=\mathcal{D}^{++} \mathcal{F}_{a}^{-}=D^{++} \mathcal{F}_{a}^{-}-\frac{1}{2} \partial_{a+} \partial_{b+} L^{+4} \mathcal{F}^{b-}
$$

Substituting (3.19) and (3.36) into (3.35), we obtain,

$$
\begin{aligned}
S_{b o s} & =S_{k i n}+S_{p o t}=\frac{1}{2} \int d^{4} x_{A} g_{a i, b j}\left(x_{A}\right) \partial^{\mu} f^{a i} \partial_{\mu} f^{b j}-\int d^{4} x_{A} V\left(f^{a i}\right), \\
V\left(f^{a i}\right) & =m^{2} \int d u \partial_{a+}\left(F_{b}^{+} \lambda^{b+}\right) \Omega^{a d}\left[\mathcal{F}_{e}^{-} \partial_{d+} \lambda^{e+}+k^{c i}\left(\partial_{c i} \mathcal{F}_{d}^{-}-2 \Omega_{d e} E_{c i}^{e-}\right)\right] .
\end{aligned}
$$


The kinetic sigma model term in (3.38) has the same form as the massless bosonic action (3.22). Note that the potential in the generic case still displays a harmonic dependence while the kinetic term does not depend on the harmonic variables. The genuine scalar potential in $x$-space is obtained after performing the integration over harmonics. In Appendix B, we give the sufficient condition under which this integration can be explicitly performed.

\subsection{Massive HK sigma model in HSS: a multi-center case}

In the previous subsection we derived the component action of the general massive HK nonlinear sigma model with at least one triholomorphic isometry. We did not specify the precise realization of this isometry. We obtained the kinetic term of the nonlinear sigma model which has exactly the form prescribed in Refs. [36, 11. However, in the general case the harmonic integral in the scalar potential cannot be computed in a simple way. Fortunately, in the case of four-dimensional HK manifolds the situation is simplified radically due to the theorem [40, 35] claiming that any 4-dimensional HK metric with at least one $U(1)$ triholomorphic isometry falls into the class of Gibbons-Hawking multi-center metrics [41]. Moreover, it can be shown (see 29] and refs. therein) that the HK potentials for such metrics can always be brought to the form $L_{m c}^{+4}=L^{+4}\left(u^{+} \cdot q^{+}, u\right)$ where $u^{+} \cdot q^{+}=u^{+a} q_{a}^{+}$and the isometry is realized as the shift $q^{+a} \rightarrow q^{+a}+\varepsilon u^{+a}$. As a result, the computation of the potential is drastically simplified.

In the present case we have

$$
\lambda^{a+}=u^{+a}, \quad \frac{\partial}{\partial x^{5}} q^{a+}=m u^{+a} .
$$

In this particular case, the Lagrangian in (3.35) can be rewritten as follows

$$
\begin{aligned}
\mathcal{L} & =\mathcal{L}_{\text {kin }}+\mathcal{L}_{\text {pot }}, \\
\mathcal{L}_{\text {kin }} & =-\frac{1}{2} \int d u A_{\mu}^{a-} \partial^{\mu} F_{a}^{+}, \\
\mathcal{L}_{\text {pot }} & =-i m \int d u M^{a-} u_{a}^{+},
\end{aligned}
$$

where we used $M_{a}^{-}=-N_{a}^{-}$, which follows from (3.33) and (3.34).

Our purpose is to derive the component action of physical bosons in $x$-space from the $(x, u)$ space action (3.41). One of the ways to obtain it is to substitute (3.40) into the general formula 
(3.38). However, it is easier to proceed directly by solving eqs. (3.11), (3.14), (3.33) and (3.34). We carry out this in two steps. First, we solve the equations of motion (3.11) and (3.14), and derive the kinetic term. As a result of solving these equations, $F_{a}^{+}$and $A_{a}^{\mu-}$ are expressed in terms of the dynamical physical fields $f^{a i}$. It turns out that it is actually enough to solve equation for $A_{a}^{\mu-}$ partially, as distinct from the equation for $F_{a}^{+}$which should be solved exactly. Secondly, we solve the equations (3.33) and (3.34). These solutions are needed to derive the scalar potential. We will see that the scalar potential is expressed in terms of the analytic HK potential after substituting the solutions into (3.43).

The equations of motion (3.11) and (3.14) are written in the considered particular case as

$$
\begin{aligned}
& D^{++} F_{a}^{+}-u_{a}^{+} L^{+2}=0, \\
& D^{++} A_{a}^{\mu-}-u_{a}^{+}\left(u^{+} \cdot A^{\mu-}\right)-2 \partial^{\mu} F_{a}^{+}=0,
\end{aligned}
$$

where

$$
\begin{aligned}
& L^{+2}\left(u^{+} \cdot F^{+}, u\right)=-\frac{1}{2} \frac{\partial L^{+4}}{\partial\left(u^{+} \cdot F^{+}\right)}, \\
& L\left(u^{+} \cdot F^{+}, u\right)=-\frac{1}{2} \frac{\partial^{2} L^{+4}}{\partial\left(u^{+} \cdot F^{+}\right)^{2}} .
\end{aligned}
$$

First we solve (3.44). Substituting the following ansatz

$$
F^{a+}=f^{a i} u_{i}^{+}+v^{a+}\left(f^{a i}, u\right)
$$

into (3.44), we obtain

$$
\partial^{++} v^{a+}\left(f^{a i}, u\right)=u^{+a} L^{+2}
$$

Up to a gauge freedom, $v^{a+}(f)$ can be written as

$$
v^{a+}\left(f^{a i}, u\right)=u^{+a} v\left(f^{a i}, u\right)
$$

Indeed,

$$
u^{+} F^{+}=u^{+a}\left(f_{a}^{i} u_{i}^{+}+u_{a}^{+} v\left(f^{a i}, u\right)\right)=u^{+a} f_{a}^{i} u_{i}^{+} .
$$

Thus, eq. (3.48) amounts to

$$
\partial^{++} v\left(f^{a i}, u\right)=L^{+2}\left(u^{+} \cdot f^{+}, u\right) .
$$


Using the harmonic Green function [29], we obtain the general solution of this equation as

$$
v\left(f^{a i}, u\right)=\int d w \frac{u^{+} \cdot w^{-}}{u^{+} \cdot w^{+}} L^{+2}\left(w^{+} \cdot f^{+}, w\right) .
$$

To be convinced that this is indeed a solution, one should substitute it into (3.51) and use

$$
\partial_{u}^{++}\left(\frac{u^{+} \cdot w^{-}}{u^{+} \cdot w^{+}}\right)=\delta^{(2,-2)}(u, w)
$$

Thus, we find the final form of the solution of (3.44) to be

$$
F^{a+}=f^{a i} u_{i}^{+}+u^{+a} \int d w \frac{u^{+} \cdot w^{-}}{u^{+} \cdot w^{+}} L^{+2}\left(w^{+} \cdot f^{+}, w\right)
$$

which will be used for computing the kinetic term.

Next, we partially solve eq. (3.45). Multiplying (3.45) by $u_{a}^{+}$and $u_{a}^{-}$, we obtain

$$
\begin{aligned}
& D^{++}\left(u^{+} \cdot \tilde{A}_{\mu}^{-}\right)=0, \\
& D^{++}\left(u^{-} \cdot \tilde{A}_{\mu}^{-}\right)-\left(u^{+} \cdot \tilde{A}_{\mu}^{-}\right)(1-L)+2 u^{+a} \partial_{\mu} f_{a}{ }^{i} u_{i}^{-} L+2 \partial_{\mu} v\left(f^{a i}, u\right)=0,
\end{aligned}
$$

where

$$
\tilde{A}_{a}^{\mu-}=A_{a}^{\mu-}-2 \partial^{\mu} f_{a}^{i} u_{i}^{-} .
$$

Eq. (3.55) implies that $B^{\mu}(x) \equiv\left(u^{+} \cdot \tilde{A}^{\mu-}\right)$ does not depend on the harmonics. Substituting this into (3.56) and taking the harmonic integral of the l.h.s. of (3.56), we find

$$
B^{\mu}(x)=-\frac{2}{1+V_{0}} \partial^{\mu} f^{a i} V_{a i}
$$

where

$$
\begin{aligned}
V_{a i} & =\int d u u_{a}^{+} u_{i}^{-} L\left(u^{+} \cdot f^{+}, u\right), \\
V_{0} & =\epsilon^{a i} V_{a i}=-\int d u L\left(u^{+} \cdot f^{+}, u\right) .
\end{aligned}
$$

Here, we have used the property

$$
\int d u v(f, u)=\int d u d w \frac{u^{+} \cdot w^{-}}{u^{+} \cdot w^{+}} L^{+2}\left(w^{+} \cdot f^{+}, w\right)=0,
$$

which can be proved by representing $u_{i}^{+}$in the numerator of the integrand as $u_{i}^{+}=\partial_{u}^{++} u_{i}^{-}$, integrating by parts with respect to $\partial_{u}^{++}$and using the properties

$$
\left(u^{-} \cdot w^{-}\right) \delta^{(1,-1)}(u, w)=0
$$


and

$$
\int d u \partial^{++} f^{(q)}(u)=0
$$

where $q$ is a $U(1)$ charge. From (3.57) and (3.58), we obtain

$$
A_{\mu a}^{-}=-\left[u_{a}^{+}\left(u^{-} \cdot A_{\mu}^{-}\right)+2 u_{a}^{-}\left(\partial_{\mu} f^{b j} u_{b}^{+} u_{j}^{-}+\frac{1}{1+V_{0}} \partial_{\mu} f^{b j} V_{b j}\right)\right] .
$$

Now we are ready to compute the kinetic term. As already mentioned, in order to compute the metric, there is no need to explicitly solve eq. (3.56) for the remaining unknown $\left(u^{-} \cdot A_{\mu}^{-}\right)=$ $\left(u^{-} \cdot \tilde{A}_{\mu}^{-}\right)-2 \partial_{\mu} f^{a i} u_{a}^{-} u_{i}^{-}$. We substitute (3.54) and (3.62) into (3.42) and, integrating by parts with respect to $D^{++}$, obtain

$$
\begin{aligned}
\mathcal{L}_{k i n}= & -\frac{1}{2} \int d u A_{\mu}^{a-} \partial^{\mu} F_{a}^{+} \\
= & \int d u\left\{\frac{1}{2} D^{++}\left(u^{-} \cdot A_{\mu}^{-}\right) \partial^{\mu} f^{a i} u_{(a}^{+} u_{i)}^{-}\right. \\
& \left.-\left(\partial_{\mu} f^{a i} u_{a}^{+} u_{i}^{-}+\frac{1}{1+V_{0}} \partial_{\mu} f^{a i} V_{a i}\right)\left(\partial^{\mu} f^{b j} u_{b}^{-} u_{j}^{+}+\partial^{\mu} v\right)\right\} .
\end{aligned}
$$

At this step, eq. (3.56) must be taken into account. We make use of it in the first term on the r.h.s. of (3.63), then perform the harmonic integral, integrate a few times by parts and use eqs. (3.61) and (3.51). Finally, we obtain the kinetic sigma model term just in the form (3.22) with

$$
g_{a i, b j}=\left(1+V_{0}\right) \epsilon_{a b} \epsilon_{i j}+V_{a i} \epsilon_{b j}+V_{b j} \epsilon_{a i}+\frac{2}{1+V_{0}} V_{a i} V_{b j} .
$$

The same metric has been earlier derived from the HSS approach in [38, 29]. There, the nonLagrangian approach was used, with the inverse metric as the basic outcome:

$$
g^{a i, b j}=\frac{1}{1+V_{0}}\left(\epsilon^{a b} \epsilon^{i j}+V^{a i} \epsilon^{b j}+V^{b j} \epsilon^{a i}+V^{2} \epsilon^{a i} \epsilon^{b j}\right)
$$

where $V^{2}=V^{a i} V_{a i}$. The Lagrangian approach used above is simpler and more direct. It can be easily employed to find the explicit form of the scalar potential term in (3.38) for the considered multi-center metrics.

To this end, we should still solve eqs. (3.33) and (3.34) which in this particular case have the following form

$$
\begin{aligned}
& \mathcal{D}^{++} M_{a}^{-}+i m u_{a}^{+}=0, \\
& \mathcal{D}^{++} N_{a}^{-}-i m u_{a}^{+}=0 .
\end{aligned}
$$


Thus eqs. (3.66) and (3.67) are reduced to the single equation

$$
D^{++} M_{a}^{-}-u_{a}^{+}\left(u^{+} \cdot M^{-}\right) L+i m u_{a}^{+}=0
$$

Introducing

$$
\tilde{M}^{a-}=M^{a-}+i m u^{-a}
$$

and projecting (3.68) on the harmonics $u_{a}^{+}$and $u_{a}^{-}$, respectively, we obtain

$$
\begin{aligned}
& D^{++}\left(u^{+} \cdot \tilde{M}^{-}\right)=0 \\
& D^{++}\left(u^{-} \cdot \tilde{M}^{-}\right)-\left(u^{+} \cdot \tilde{M}^{-}\right)(1-L)-i m L=0 .
\end{aligned}
$$

It follows from (3.70) that $\left(u^{+} \cdot \tilde{M}^{-}\right)$does not depend on harmonics,

$$
\left(u^{+} \cdot \tilde{M}^{-}\right)=A(x) .
$$

Substituting this into (3.71) and integrating the 1.h.s. of the latter over harmonics, we obtain

$$
A(x)=i m \frac{V_{0}}{1+V_{0}} \quad \Rightarrow \quad\left(u^{+} M^{-}\right)=A-i m=-i m \frac{1}{1+V_{0}} .
$$

Substituting (3.73) into (3.43), we find the final form of the scalar potential to be

$$
\mathcal{L}_{\text {pot }}(x, u)=-m^{2} \frac{1}{1+V_{0}}, \quad \partial^{++} \mathcal{L}_{\text {pot }}=0 .
$$

Thus, we have managed to solve the equations of motion (3.11), (3.14), (3.33) and (3.34) and so have found the explicit form of the component bosonic action (3.38) for the multi-center case. Both the HK metric and potential term in (3.38) are expressed, by eqs. (3.64) and (3.74), in terms of the single object, multi-center potential $V_{0}$ defined in (3.60).

Let us derive the same result (3.74) in another way. Applying the general formula (3.27) to the particular case (3.40) and taking into account that it follows from eq. (3.54) that $\delta f^{a i}=$ $\varepsilon \epsilon^{a i} \Rightarrow k^{a i}=\epsilon^{a i}$, we find

$$
u^{+a}=-E_{b i}^{a+} k^{b i}=-E_{b i}^{a+} \epsilon^{b i}=\mathcal{D}^{++} E_{b i}^{a-} \epsilon^{b i}
$$

Then we can rewrite the Lagrangian (3.43) as

$$
\mathcal{L}_{p o t}=-i m M^{a-} \Omega_{a c} E_{b i}^{c+} \epsilon^{b i}
$$


Moreover, it is easy to find

$$
M^{a-}=-i m E_{b i}^{a-} \epsilon^{b i}
$$

Now we substitute this into (3.76), use the definition (3.23) and take into account eq. (3.21) and the fact that under the $u$-integral one can integrate by parts. As the result we obtain

$$
\mathcal{L}_{\text {pot }}=-\frac{m^{2}}{2} g_{a i, b k} \epsilon^{a i} \epsilon^{b k}=-\frac{m^{2}}{2} g_{a i, b k} k^{a i} k^{b k},
$$

which is just the square of the norm of Killing vector $k^{a i}=\epsilon^{a i}$. Using (3.64), we find

$$
g_{a i, b k} \epsilon^{a i} \epsilon^{b k}=\frac{2}{1+V_{0}},
$$

i.e., we come back to the expression (3.74). This means that the scalar potential is determined by the norm of the Killing vector. This fact was originally obtained in [14] by means of an on-shell formalism.

Finally, let us show that eqs. (3.64) and (3.74) can be put in the standard multi-center form. Introducing,

$$
\vec{V}=-\frac{i}{2}(\vec{\tau})^{a i} V_{a i}, \quad \vec{X}=\frac{i}{\sqrt{2}}(\vec{\tau})^{a i} f_{a i}, \quad \varphi=\frac{1}{\sqrt{2}} \epsilon_{a i} f^{a i}, \quad U=1+V_{0},
$$

where $\vec{\tau}^{a i}$ are the Pauli matrices, ${ }^{4}$ we can write down Lagrangian (3.41) in the form

$$
\mathcal{L}=\frac{1}{2}\left\{U \partial_{\mu} \vec{X} \cdot \partial^{\mu} \vec{X}+U^{-1} \mathcal{D}_{\mu} \varphi \mathcal{D}^{\mu} \varphi-m^{2} U^{-1}\right\}
$$

where $m$ has been changed by $m / \sqrt{2}$, and $\vec{X}=\left(X^{1}, X^{2}, X^{3}\right), \varphi$ are real scalar fields, and $\mathcal{D}_{\mu} \varphi=\partial_{\mu} \varphi+\vec{V} \cdot \partial_{\mu} \vec{X}$. The fields $\vec{V}$ and $1+V_{0}$, by their definition, satisfy the differential equations

$$
\vec{\nabla} \times \vec{V}=\vec{\nabla} U, \quad \Delta U=0, \quad \frac{\partial}{\partial \varphi}\left(1+V_{0}\right)=0
$$

The scalar potential is given by

$$
V=m^{2} U^{-1}
$$

\footnotetext{
${ }^{4}$ Here $\vec{\tau}^{a i}=\epsilon^{a b} \vec{\tau}_{b}^{i}$ where $\vec{\tau}_{a}^{i}$ are the standard Pauli matrices.
} 
This precisely coincides with what has been found in [14]. In this parameterization, the $U(1)$ isometry (3.40) is realized as a shift of the coordinate $\varphi$ of the HK manifold:

$$
\delta \varphi=\sqrt{2} \varepsilon, \quad \delta \vec{X}=0 .
$$

Note that it is possible to extend the above consideration to the case of $4 n$ dimensional HK manifolds whose metric have $n$ commuting translation isometries, i.e., to the general case of toric HK metrics. In this case, the ansatz for the analytic HK potential is $L_{\text {toric }}^{+4}=L^{+4}\left(u^{+i} q_{a i}^{+}, u\right)$ where $a=1, \cdots n[29$.

Now we consider two particular examples.

i) Taub-NUT case

The analytic potential in the Taub-NUT (TN) case can be chosen as ${ }^{5}$

$$
L_{T N}^{+4}\left(u^{+} \cdot q^{+}, u\right)=\frac{2}{\lambda}\left(g^{++}\right)^{2}=\frac{2}{\lambda}\left(\frac{L^{++}-c^{++}}{1+\sqrt{1+\left(L^{++}-c^{++}\right) c^{--}}}\right)^{2}, \quad L^{++}=u^{+} \cdot q^{+}
$$

where $c^{ \pm \pm}=c^{i j} u_{i}^{ \pm} u_{j}^{ \pm}, c^{2}=\frac{1}{2} c^{i j} c_{i j}=1$ and $\lambda$ is a constant. The corresponding potential $V_{0}$ is given by

$$
V_{0}=\frac{1}{2} \int d u \frac{\partial^{2} L_{T N}^{+4}}{\partial\left(L^{++}\right)^{2}}=\frac{1}{2 \lambda} \int d u \frac{1}{\left[1+\left(L^{++}-c^{++}\right) c^{--}\right]^{3 / 2}} .
$$

To compute the harmonic integral in (3.86), we make use of the general formula derived in Ref. [42]:

$$
\int d u \frac{1}{\left[1+\left(G^{++}-c^{++}\right) c^{--} / c^{2}\right]^{3 / 2}}=\frac{\sqrt{c^{i k} c_{i k}}}{\sqrt{G^{i k} G_{i k}}}
$$

where

$$
G^{++}=G^{i k} u_{i}^{+} u_{k}^{+}
$$

Using this general formula and choosing the particular $S U(2)_{R}$ frame, $c^{11}=c^{22}=0, c^{12}=i$, it is easy to find

$$
U_{T N}=1+V_{0}=1+\frac{1}{\sqrt{2} \lambda} \frac{1}{\sqrt{L^{i k} L_{i k}}} .
$$

\footnotetext{
${ }^{5}$ The form of the TN and Eguchi-Hanson (EH) HK potentials can be found in Chapter 6.6.1 of Ref. 29] (see eqs. (6.72) and (6.73) there). The HK potential for the double Taub-NUT metric is obtained by a slight modification of these potentials.
} 
We can rewrite (3.89) in terms of the multi-center coordinate $\vec{X}$. Using the relations

$$
\begin{gathered}
L^{++}=u^{+a} F_{a}^{+}=-f^{a i} u_{a}^{+} u_{i}^{+}=-f^{(a i)} u_{(a}^{+} u_{i)}^{+}, \\
\vec{X} \cdot \vec{X}=f_{(a i)} f^{(a i)},
\end{gathered}
$$

we find

$$
U_{T N}=1+\frac{1}{\sqrt{2} \lambda} \frac{1}{|\vec{X}|}
$$

This form of the one-center TN potential corresponds to the center located at $\vec{X}=0$. In the following example, for later convenience, we choose another position of the center.

ii) Double Taub-NUT case

Now we consider more general double Taub-NUT (DTN) case. The relevant analytic HK potential reads

$$
\begin{aligned}
L_{D T N}^{+4}\left(u^{+} \cdot q^{+}, u\right)= & 2\left(\frac{L^{++}}{1+\sqrt{1+L^{++} \eta^{--}}}\right)^{2}+\frac{2}{\gamma}\left(\frac{L^{++}}{1+\sqrt{1-L^{++} \eta^{--}}}\right)^{2} \\
& -(1-a)\left(L^{++}\right)^{2},
\end{aligned}
$$

where $a$ and $\gamma$ are some constants and $\eta^{ \pm \pm}=\eta^{i k} u_{i}^{ \pm} u_{i}^{ \pm}$. If $\eta^{i k}=0$ we return to the TN case. For $\eta^{i k} \neq 0$, one can always choose $\eta^{2}=\frac{1}{2} \eta^{i j} \eta_{i j}=1$ by the appropriate rescaling of $q^{a+}$. As we will see, $\eta^{i j}$ specifies the location of the centers. The potential $V_{0}$ in the present case reads

$$
V_{0}=\frac{1}{2} \int d u\left(\frac{1}{\left[1+L^{++} \eta^{--}\right]^{3 / 2}}+\frac{1}{\gamma} \frac{1}{\left[1-L^{++} \eta^{--}\right]^{3 / 2}}\right)-(1-a),
$$

whence

$$
U_{D T N}=1+V_{0}=a+\frac{1}{\sqrt{2}} \frac{1}{\sqrt{\left(L^{i k}+\eta^{i k}\right)\left(L_{i k}+\eta_{i k}\right)}}+\frac{1}{\sqrt{2} \gamma} \frac{1}{\sqrt{\left(L^{i k}-\eta^{i k}\right)\left(L_{i k}-\eta_{i k}\right)}} .
$$

Introducing,

$$
\vec{\xi}=\frac{i}{\sqrt{2}} \vec{\tau}^{i j} \eta_{i j},
$$

and using (3.90), we can rewrite (3.94) as

$$
U_{D T N}=a+\frac{1}{\sqrt{2}} \frac{1}{|\vec{X}+\vec{\xi}|}+\frac{1}{\sqrt{2} \gamma} \frac{1}{|\vec{X}-\vec{\xi}|} .
$$


For $a \neq 0$ this is a two-center ALE potential, with the constant vector $\vec{\xi}$ specifying the position of both centers (they are collinear to each other). For $a=0$, the potential $U_{D T N}$ becomes the general EH potential with non-equal "masses". Like the DTN potential itself, its EH limiting case possesses only $U(1) \times U(1)$ isometry in contrast to the $S U(2) \times U(1)$ isometry of the standard EH potential which is recovered under the choice $\gamma=1$.

\section{Structure of SUSY vacua and the BPS equation}

From the form of the scalar potential (3.74) we can find the SUSY vacuum condition which is similar to that in the $\mathcal{N}=1$ case. The condition of SUSY vacuum is the vanishing of the scalar potential

$$
0=V\left(f^{a i}\right)=\frac{m^{2}}{1+V_{0}}=g_{a i, b j} k^{a i} k^{b j} .
$$

We find that in our parameterization of the multi-center case the SUSY vacuum exists, provided there is a point where the potential $V_{0}$ goes to infinity,

$$
V_{0}=\frac{1}{2} \int d u \frac{\partial^{2} L^{+4}}{\partial\left(L^{++}\right)^{2}} \rightarrow \infty .
$$

The condition (4.2) is the $\mathcal{N}=2$ multi-center counterpart of the general SUSY vacuum conditions (2.9) and (2.10) of the $\mathcal{N}=1$ theory. We expect that this condition imposes strong restrictions on the original HK potential $L^{+4}$, though for the time being we do not know them in full generality. Now we apply eq. (4.2) to the previous examples.

i) $T N$ case

In the $\mathrm{TN}$ case, it follows from (3.91) that the condition (4.2) can be realized only for the vacuum expectation value $\vec{X}=0$. Thus the theory has only one SUSY vacuum. As a consequence, no domain wall solution can be found in this case since the existence of the domain wall solutions requires that the theory has at least two vacua.

ii) DTN case

In the DTN case, the theory has two discrete vacua which are realized at vacuum expectation

values $\vec{X}=-\vec{\xi}$ and $\vec{X}=\vec{\xi}$. Indeed, in this case there exists the domain wall solution as we shall see soon. 


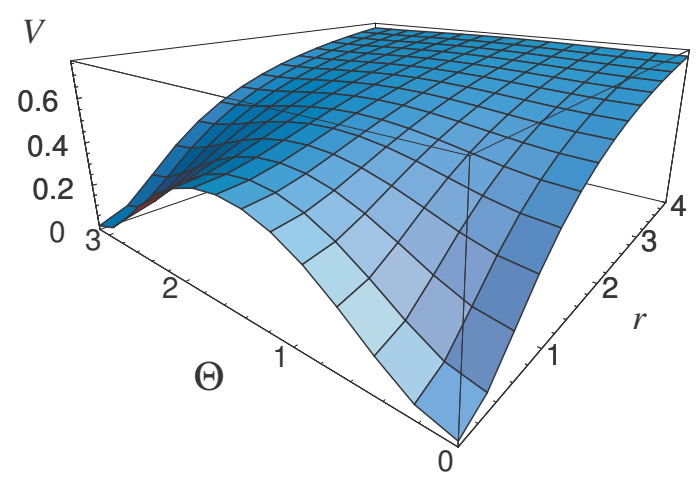

(a) 3D Plot of the potential.

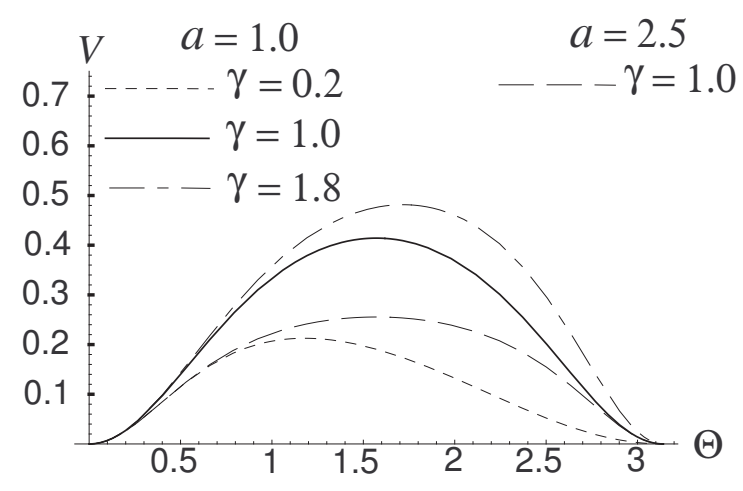

(b) Plot of the potential along $\Theta$ axis.

Figure 1: Plots of the potential for $\xi=1.0$ and $m=1.0$.

In order to find the behaviour of the potential in the DTN case, we take $\vec{\xi}=(0,0, \xi)$ and introduce the spherical coordinates such as

$$
X^{1}=r \sin \Theta \cos \Psi, \quad X^{2}=r \sin \Theta \sin \Psi, \quad X^{3}=\sqrt{r^{2}+\xi^{2}} \cos \Theta, \quad \varphi=\Phi+\Psi
$$

In this parameterization, the geometrical meaning of the target manifold becomes clear: the fields $r$ and $\Theta$ are the coordinates of base manifold $S^{2}$ and $\Phi$ and $\Psi$ form a fiber over this base manifold. The DTN potential (3.96) takes the following form in the coordinates (4.3):

$$
U_{D T N}=a+\frac{1}{\sqrt{2}}\left(\frac{1}{\left|\sqrt{r^{2}+\xi^{2}}+\xi \cos \Theta\right|}+\frac{1}{\gamma} \frac{1}{\left|\sqrt{r^{2}+\xi^{2}}-\xi \cos \Theta\right|}\right) .
$$

Note that the potential (4.4) depends only on the real coordinates $r$ and $\Theta$, and not on $\Phi$ and $\Psi$, which reflects the presence of $U(1) \times U(1)$ isometry. So the vacuum configurations "live" on the submanifold $S^{2}$ of the full target space.

The behaviour of the scalar potential in the spherical reparameterization for different ranges of the involved parameters is shown in Fig. 1. Fig. 1-(a) shows the 3D plot of the scalar potential. It is seen that there are two discrete vacua at $(r, \Theta)=(0,0)$ and $(0, \pi)$. Fig. 1-(b) shows the plot of the potential along $\Theta$ axis for some values of $a$ and $\gamma$. For $\gamma=1$ the shape of the potential is symmetric with respect to $\Theta=\pi / 2$ axis. As the value of $\gamma$ deviates from $\gamma=1$, the scalar potential becomes asymmetric. If $\gamma$ goes to infinity, the vacuum is realized only at the single 
point $(r, \Theta)=(0,0)$ since the third term in eq. (3.96) disappears and the potential (3.96) reduces to the one of the TN case (with an arbitrary constant $a$ ). As a increases, the behaviour becomes gentle, i.e., the value of the potential at any value of $\Theta$ excepting vacua decreases.

The vacuum expectation values, for instance, $\vec{X}=0$ in the $\mathrm{TN}$ case, can be easily cast in the HSS language using (3.90); these amount to $L^{++}=0$ in the TN case and to $L^{++}=-\eta^{++}$ and $L^{++}=\eta^{++}$in the DTN case. For these special values of $L^{++}$, the analytic potentials (3.85) and (3.92), equally as their second derivatives entering (3.86) and (3.93), acquire singularities at some points of the harmonic sphere $S^{2} \sim\left\{u_{i}^{+}, u_{k}^{-}\right\}$. As the result, the $u$-integral (4.2) which specifies the sufficient condition for vacuum to be the SUSY one becomes divergent. E.g., in the TN case, substituting $L^{++}=0\left(L^{i j}=0\right)$ into (4.2), one obtains harmonic integral

$$
\left.V_{0}\right|_{L^{i j=0}}=\frac{1}{2} \int d u \frac{1}{\left[1-c^{++} c^{--}\right]^{3 / 2}}
$$

which is divergent. The same divergent integrals are obtained in the DTN case for two values of $L^{++}$, namely for $L^{++}=-\eta^{++}$and $L^{++}=\eta^{++}$. The existence of two discrete vacua in the latter case guarantees the existence of the domain wall solution.

In the following, we consider the general BPS equation and apply it to the DTN case. If we assume that there is a non-trivial configuration along the spatial $y$ direction and this configuration is static, the energy density can be written as

$$
\begin{aligned}
\mathcal{E} & =\frac{1}{2} U \partial_{2} \vec{X} \cdot \partial_{2} \vec{X}+\frac{1}{2} U^{-1} \mathcal{D}_{2} \varphi \mathcal{D}_{2} \varphi+\frac{1}{2} m^{2} U^{-1} \\
& =\frac{1}{2} U\left(\partial_{2} \vec{X}-m U^{-1} \vec{n}\right) \cdot\left(\partial_{2} \vec{X}-m U^{-1} \vec{n}\right)+\partial_{2} \vec{X} \cdot \vec{n}+\frac{1}{2} U^{-1} \mathcal{D}_{2} \varphi \mathcal{D}_{2} \varphi \\
& \geq \partial_{2} \vec{X} \cdot \vec{n},
\end{aligned}
$$

where $\vec{n}$ is a unit vector. BPS equation is easily read off as

$$
\begin{gathered}
\partial_{2} \vec{X}-m U^{-1} \vec{n}=0, \\
\mathcal{D}_{2} \varphi=0
\end{gathered}
$$

Taking $\vec{n}=(0,0,1)$ and using $\vec{n} \cdot \vec{V}=0$ [4], BPS equation is simplified to

$$
\begin{aligned}
& \frac{\partial \varphi}{\partial y}=0, \quad \frac{\partial X^{1}}{\partial y}=\frac{\partial X^{2}}{\partial y}=0 \\
& \frac{\partial X^{3}}{\partial y}=m U^{-1}
\end{aligned}
$$




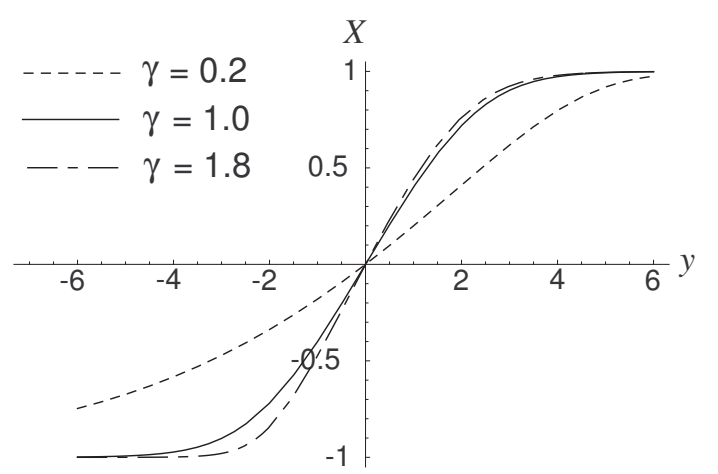

(a)Wall solutions for some values of $\gamma$ with $a=1$.

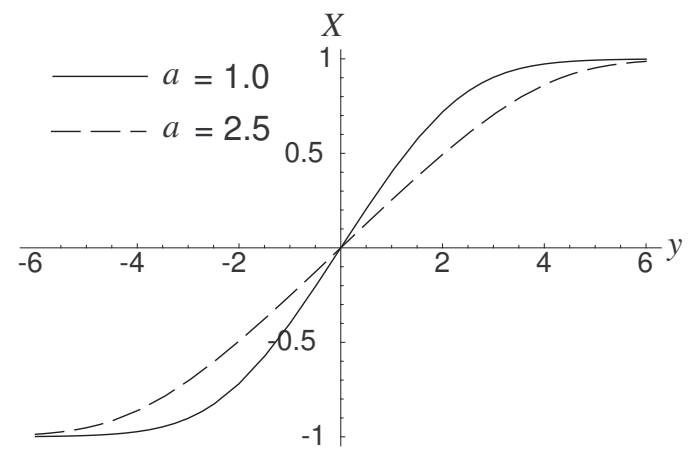

(b) Wall solutions for some values of $a$ with $\gamma=1$.

Figure 2: Behaviour of the domain wall solution. The wall is positioned at $y=0$.

Eq. (4.9) can be easily solved by

$$
\varphi=\text { const }, \quad X^{1}=\text { const }, \quad X^{2}=\text { const }
$$

Without loss of generality, these constants can be put equal to zero, i.e., $\varphi=X^{1}=X^{2}=0$. Using these solutions and substituting (3.96) into (4.10), we bring eq. (4.10) to the form

$$
\frac{\partial X}{\partial y}=m \frac{\sqrt{2} \gamma\left(\xi^{2}-X^{2}\right)}{\sqrt{2} a \gamma\left(\xi^{2}-X^{2}\right)+\gamma(\xi-X)+\xi+X}, \quad X \equiv X^{3} .
$$

This equation can be easily solved. Fig. 2 shows the profiles of the domain wall solutions. Fig. 2-(a) shows the profiles for some values of $\gamma$ with $a=1$. For $\gamma=1$, the metric becomes the DTN metric with equal masses and the scalar configuration is symmetric at the center of the wall for $y=0$. In particular, for $\gamma=1$ and $a=0$ (the standard EH case), analytic solution is obtained as

$$
X=\xi \tanh \left(\frac{\sqrt{2} m}{2 \xi}\left(y+y_{0}\right)\right)
$$

where $y_{0}$ is an integration constant which specifies the position of the wall. As $\gamma$ deviates from the value $\gamma=1$, the scalar configuration becomes asymmetric. For fixed $\gamma$, the behaviour of the solution is shown in Fig. 2-(b). As was mentioned, when $\gamma \rightarrow \infty$, the metric approaches the TN metric and therefore the domain wall solution does not exist in this limit. 


\section{$5 \quad$ Summary and concluding remarks}

In this paper we have studied $\mathcal{N}=2$ massive nonlinear sigma model starting from the action in the off-shell HSS formulation which manifests the full $\mathcal{N}=2$ SUSY. The scalar potential was obtained by assigning to $q^{+}$a dependence on the central charge coordinate $x_{5}$, such that $\partial / \partial x_{5}$ is identified with the Killing vector of the isometry. The component bosonic action was obtained based on the one-to-one correspondence between the Lagrangian and non-Lagrangian approaches to the HK geometry. As was shown in 29], the kinetic term at the component level in the general nonlinear sigma model is composed of the vielbeins and has a form which is independent of the harmonic variables. On the other hand, the scalar potential in the general case of one isometry still involves an integration over harmonics. Its more preferable form, which does not contain the harmonic integral, is derived in the Appendix B. It is shown there that the integration over harmonic variables can be explicitly performed under the particular condition which is satisfied, e.g., in the multi-center case.

Massive nonlinear sigma models with multi-center metrics were examined. In the generic HK case, solving the kinematical part of the equations of motion is very difficult problem. However, in the multi-center case, the situation is much simpler. We solved the kinematical part of the equations of motion and obtained the physical component action where integration over harmonic variables was performed to the end. It was shown that both the target metric and the scalar potential can be expressed in terms of the single analytic HK potential. The scalar potential was found to be fully specified by the norm of the Killing vector, which is in agreement with the earlier derivation of Ref. [14. Given the explicit form of the scalar potential, we discussed the SUSY vacuum condition. The SUSY vacuum condition was related to the analytic HK potential. This result is the $\mathcal{N}=2$ extension of the similar condition in $\mathcal{N}=1$ theory which involves the Kähler potential and the superpotential. We derived BPS equation in the general multi-center case and BPS domain wall solution was obtained for the DTN case.

We would like to conclude by mentioning some directions for future work.

It would be interesting to extend our discussion of the multi-center four-dimensional case to the general case of toric HK manifolds, i.e., $4 n$ dimensional HK manifolds with $n$ commuting triholomorphic isometries. 
In this paper, we studied the nonlinear sigma model without explicit use of the quotient construction, starting from the given HK potential $L^{+4}(q, u)$ (though both the TN and DTN HK potentials can actually be deduced from the appropriate HSS version of the quotient construction, see [28, 44, 45]). It is interesting to discuss the massive nonlinear sigma model with isometries like the models we considered here within the quotient construction. We expect essential simplifications in this regard. In particular, the quotient method could make easier coupling of the massive nonlinear sigma model to the supergravity, which is also an interesting task. In this case, instead of HK sigma models we would have sigma models with quaternion-Kähler (QK) target manifolds [46]. The HSS formulation of the $\mathcal{N}=2, d=4$ supergravity and massless QK sigma models in the supergravity background was worked out in Refs. [47, 48, 44]. It would be tempting to extend it to the case of massive QK sigma models.

\section{Acknowledgements}

The collaboration with M. Naganuma, M. Nitta and N. Sakai motivated M.A. to start this work. The work of E.I. was supported in part by grants DFG No.436 RUS 113/669, RFBRDFG 02-02-04002, INTAS 00-00254, RFBR 03-02-17440 and grants of the Heisenberg-Landau and Votruba-Blokhintsev programs. He thanks the Institute of Physics in Prague for warm hospitality at the initial stage of this study.

\section{A Relation between the Killing vectors in harmonic and ordinary spaces}

In this appendix, we present the expression of the Killing vector $k^{a i}$ in terms of the central basis Killing potential.

In order to obtain this expression, we use the formulas for vielbeins, which were derived in the non-Lagrangian geometric approach [38. Firstly, we multiply both sides of the relation in (3.27),

$$
\lambda^{a+}=-E_{c k}^{a+} k^{c k}=\frac{1}{2} \Omega^{a b} \partial_{b+} f^{c i} \partial_{c i} \Lambda^{k l} u_{k}^{+} u_{l}^{+}
$$


by $E_{a}^{-d k}$ which is the inverse to the vielbein $E_{d k}^{a+}$. Then we use the representation 38 ]

$$
E_{b}^{-c i}=-\partial_{b+} f^{c i}+E_{b}^{-d-} \partial_{d-} f^{c i}
$$

where $\partial_{d-}=\partial / \partial F^{d-}$ is the derivative with respect to the non-analytic coordinate $F^{d-}$ defined in (3.18), and the precise form of $E_{b}^{-d-}$ is of no interest for our purpose. Due to the analyticity of $\Lambda^{++}$, i.e. the property $\partial_{d-} \Lambda^{++}=0$, the result of multiplication of (3.27) by $E_{a}^{-d k}$ can be written as

$$
E_{a}^{-d k} E_{b i}^{a+} k^{b i}=\frac{1}{2} \Omega^{a b} E_{a}^{-d k} E_{b}^{-c i} \partial_{c i} \Lambda^{l n} u_{l}^{+} u_{n}^{+}
$$

Next we make use of eq. (3.21) and the relations

$$
\begin{aligned}
& E_{a}^{-d k} E_{b i}^{a+}+E_{a}^{+d k} E_{b i}^{a-}=\delta_{b}^{d} \delta_{i}^{k} \\
& E_{a}^{+d k}=-\mathcal{D}^{++} E_{a}^{-d k}
\end{aligned}
$$

After that, integrating both sides of (A.3) over harmonics with making use of (A.4), (A.5) and (3.21) and taking into account that the $S p(n)$ connections drop out altogether because all $S p(n)$ indices are contracted, we obtain

$$
k^{a i}=\int d u \Omega^{c d}\left(E_{c}^{-a i} E_{d}^{-b j}\right) \partial_{b j} \Lambda^{k l} u_{k}^{+} u_{l}^{+} .
$$

Using the definition of the triplet of complex structures

$$
I^{ \pm \pm[a i, b j]}=I_{k l}^{[a i, b j]} u^{ \pm k} u^{ \pm l}=E_{c}^{ \pm a i} E_{d}^{ \pm b j} \Omega^{c d}
$$

and performing the harmonic integration in (A.6), we obtain the final relation

$$
k^{a i}=-\frac{1}{3} I_{(k l)}^{[a i, b j]} \partial_{b j} \Lambda^{(k l)}(f) .
$$

\section{B The scalar potential with arbitrary $U(1)$ isometry}

In this appendix, we solve the kinematical equations of motion (3.33) and (3.34). First of all, in order to solve them, we prove the following relation

$$
\partial_{a+}\left(F_{b}^{+} \lambda^{b+}\right)=\partial_{a+} D^{++}\left(\mathcal{F}_{b}^{-} \lambda^{b+}\right)=\mathcal{D}^{++} \partial_{a+}\left(\mathcal{F}_{b}^{-} \lambda^{b+}\right)+\left(\partial_{a-} \mathcal{F}_{b}^{-}\right) \lambda^{b+}
$$


where $\mathcal{F}_{a}^{-}$was defined in eq. (3.37). The first equality can be proved by using the definition (3.26) and (3.37). In order to prove the second equality, one should firstly take into account that $\mathcal{F}_{b}^{-} \lambda^{b+}$ is not analytic since $\mathcal{F}_{b}^{-}$is not analytic, $\mathcal{F}_{b}^{-}=\mathcal{F}_{b}^{-}\left(F^{+}, F^{-}, u\right)$. We shall also need the property that the harmonic covariant derivative $\mathcal{D}^{++}$includes the derivative $\partial_{d_{-}}=\partial / \partial F^{d-}$ when acting on non-analytic objects [38, 29]

$$
\mathcal{D}^{++} G_{a}\left(F^{+}, F^{-}\right)=D^{++} G_{a}+F^{d+} \partial_{d-} G_{a}-\frac{1}{2} \partial_{a+} \partial_{b+} L^{+4} G^{b} .
$$

Using (B.2), as well as the relations $\partial_{a-} F^{b+}=0$ and $\partial_{a+} F^{b+}=\delta_{a}{ }^{b}$, we can prove the second equality.

Introducing $\lambda^{a-}$ by

$$
\lambda^{a+}=D^{++} \lambda^{a-}, \quad \delta F^{a-}=\varepsilon \lambda^{a-},
$$

and using the relation

$$
\mathcal{D}^{++} \partial_{[a+} \mathcal{F}_{b]}^{-}=-\Omega_{a b}+\partial_{[b-} \mathcal{F}_{a]}^{-}, \quad \partial_{[a+} \mathcal{F}_{b]}^{-} \equiv \frac{1}{2}\left(\partial_{a+} \mathcal{F}_{b}^{-}-\partial_{b+} \mathcal{F}_{a}^{-}\right),
$$

it is straightforward to transform (B.1) to

$$
\partial_{a+}\left(F_{b}^{+} \lambda^{b+}\right)=\mathcal{D}^{++}\left(\mathcal{F}_{b}^{-} \partial_{a+} \lambda^{b+}+\lambda^{b+} \partial_{b+} \mathcal{F}_{a}^{-}+\lambda^{b-} \partial_{b-} \mathcal{F}_{a}^{-}\right)-2 \lambda_{a}^{+},
$$

whence the solution to eqs. (3.33) and (3.34) is obtained as follows

$$
\begin{aligned}
M^{a-}=-N^{a-} & =i m\left(\mathcal{F}_{b}^{-} \partial_{+}^{a} \lambda^{b+}+\lambda^{b+} \partial_{b+} \mathcal{F}^{a-}+\lambda^{b-} \partial_{b-} \mathcal{F}^{a-}-2 E_{c i}^{a-} k^{c i}\right) \\
& =i m\left(\mathcal{F}_{b}^{-} \partial_{+}^{a} \lambda^{b+}+k^{c i}\left[\partial_{c i} \mathcal{F}^{a-}-2 E_{c i}^{a-}\right]\right) .
\end{aligned}
$$

In passing from (B.5) to the first line in (B.6) we used eqs. (A.1) and (A.5). Now we can substitute the solution (B.6) in (3.35) and obtain the general form of the scalar potential (3.39).

Another, perhaps more convenient representation for the scalar potential can be obtained as follows. Using the $S p(n)$ bridge $M_{b}^{a}, M^{a b} M_{b}^{c}=\Omega^{a c}$, one redefines

$$
N_{a}^{-}=M_{a}^{b} \hat{N}_{b}^{-}, E_{a}^{-b i}=M_{a}^{d} \hat{E}_{d}^{-b i},\left(\mathcal{D}^{++}\right)_{a}{ }^{c} M_{c}^{b}=0, D^{++} \hat{E}_{d}^{-b i}=\hat{E}_{d}^{+b i}, D^{++} \hat{E}_{d}^{+b i}=0
$$

and rewrites the equation for $N_{a}^{-}$as

$$
D^{++} \hat{N}_{a}^{-}=i m \hat{E}_{a}^{-b i} \partial_{b i}\left(F_{c}^{+} \lambda^{c+}\right)
$$


The last equation is solved by

$$
\hat{N}_{a}^{-}=i m \int d w \frac{1}{\left(u^{+} \cdot w^{+}\right)} \hat{E}_{a}^{-b i} \partial_{b i}\left(F_{c}^{+} \lambda^{c+}\right) .
$$

Substituting this into (3.35), we obtain the following form of the scalar potential

$$
\begin{aligned}
\mathcal{L}_{p o t} & =-\frac{i m}{2} \int d u\left(M^{a-}-N^{a-}\right) \partial_{a+}\left(F_{b}^{+} \lambda^{b+}\right) \\
& =m^{2} \int d u d w \Omega^{a b} \hat{E}_{a}^{-d i} \partial_{d i}\left(F_{c}^{+} \lambda^{c+}\right) \frac{1}{\left(u^{+} \cdot w^{+}\right)} \hat{E}_{b}^{-e k} \partial_{e k}\left(F_{f}^{+} \lambda^{f+}\right)
\end{aligned}
$$

where the first and second multipliers are evaluated at the harmonic arguments $u$ and $w$, respectively. Using the fact that the bridges drop out under the $S p(n)$ covariant contractions and the

property that the vielbeins with "hat" are linear in the appropriate harmonics, one can rewrite (B.10) as

$$
\mathcal{L}_{\text {pot }}=m^{2} I_{(s t)}^{[a i, b k]} \mathcal{F}_{[a i, b k]}^{(s t)}-\frac{m^{2}}{2} g^{a i, b k} \mathcal{F}_{a i, b k}
$$

where

$$
\begin{aligned}
& \mathcal{F}_{a i, b k}=\int d u d w \partial_{a i}\left(F_{c}^{+} \lambda^{c+}\right) \frac{\left(u^{-} \cdot w^{-}\right)}{\left(u^{+} \cdot w^{+}\right)} \partial_{b k}\left(F_{f}^{+} \lambda^{f+}\right), \\
& \mathcal{F}_{[a i, b k]}^{(s t)}=\int d u d w \partial_{a i}\left(F_{c}^{+} \lambda^{c+}\right) \frac{u^{-(s} w^{-t)}}{\left(u^{+} \cdot w^{+}\right)} \partial_{b k}\left(F_{f}^{+} \lambda^{f+}\right) .
\end{aligned}
$$

However, it is unclear for us whether these harmonic integrals can be manifestly computed in the general case. Only in the particular case, when

$$
\left(F_{a}^{+} \lambda^{a+}\right)=-\frac{1}{2}\left(F^{a+} \partial_{a+}\right) \Lambda^{++}=g \Lambda^{++}
$$

with $g$ being a constant, these integrals can be computed in terms of $\partial_{a i} \Lambda^{(k l)}$ (using the fact that $\Lambda^{++}$is bilinear in harmonics in the central basis), although we do not quote here the precise formulas. Note that this condition is trivially satisfied for the isometry (3.40).

\section{References}

[1] P. Horava and E. Witten, Nucl. Phys. B460 (1996) 506, hep-th/9510209. 
[2] N. Arkani-Hamed, S. Dimopoulos and G. Dvali, Phys. Lett. 429B (1998) 263, hep-ph/9803315; I. Antoniadis, N. Arkani-Hamed, S. Dimopoulos and G. Dvali, Phys. Lett. 436B (1998) 257, hep-ph/9804398.

[3] L. Randall and R. Sundrum, Phys. Rev. Lett. 83 (1999) 3370, hep-ph/9905221; Phys. Rev. Lett. 83 (1999) 4690, hep-th/9906064.

[4] V.A. Rubakov and M.E. Shaposhnikov, Phys. Lett. B125 (1983) 136.

[5] O. Klein, Z. Phys. 37 (1926) 895.

[6] E. Bogomol'nyi, Sov. J. Nucl. Phys. B24 (1976) 449; M.K. Prasad and C.H. Sommerfield, Phys. Rev. Lett. 35 (1975) 760.

[7] E. Witten and D. Olive, Phys. Lett. B78 (1978) 97.

[8] M. Cvetic, F. Quevedo and S. Rey, Phys. Rev. Lett. 67 (1991) 1836; M. Cvetic, S. Griffies and S. Rey, Nucl. Phys. B381 (1992) 301, hep-th/9201007; G. Dvali and M. Shifman, Nucl. Phys. B504 (1997) 127, hep-th/9611213; Phys. Lett. 396B (1997) 64, hep-th/9612128; A. Kovner, M. Shifman and A. Smilga, Phys. Rev. D56 (1997) 7978, hep-th/9706089; B. Chibisov and M. Shifman, Phys. Rev. D56 (1997) 7990, hep-th/9706141; A. Smilga and A. Veselov, Phys. Rev. Lett. 79 (1997) 4529, hep-th/9706217; J. Edelstein, M.L. Trobo, F. Brito and D. Bazeia, Phys. Rev. D57 (1998) 7561, hep-th/9707016; V. Kaplunovsky, J. Sonnenschein and S. Yankielowicz, Nucl. Phys. B552 (1999) 209, hep-th/9811195; G. Dvali, G. Gabadadze and Z. Kakushadze, Nucl. Phys. B562 (1999) 158, hep-th/9901032; B. de Carlos and J.M. Moreno, Phys. Rev. Lett. 83 (1999) 2120, hep-th/9905165; M. Naganuma and M. Nitta, Prog. Theor. Phys. 105 (2001) 501, hep-th/0007184; D. Binosi and T. ter Veldhuis, Phys. Rev. D63 (2001) 085016, hep-th/0011113.

[9] M. Arai, S. Fujita, M. Naganuma and N. Sakai, Phys. Lett. B556 (2003) 192, hep-th/0212175; "An exact BPS wall solution in Five Dimensional Supergravity", to be published in the Proceedings of the International Workshop on "Supersymmetries and Quantum Symmetries" (SQS'03), hep-th/0311210. 
[10] M. Eto, S. Fujita, M. Naganuma and N. Sakai, "BPS multi-walls in five dimensional superravity", to be published in Phys. Rev. D68, hep-th/0306198.

[11] L. Alvarez-Gaumé and D.Z. Freedman, Comm. Math. Phys. 80 (1981) 443.

[12] J. Scherk and J.H. Schwarz, Phys. Lett. B82 (1979) 60; Nucl. Phys. B153 (1979) 61.

[13] L. Alvarez-Gaumé and D.Z. Freedman, Comm. Math. Phys. 91 (1983) 97.

[14] J.P. Gauntlett, D. Tong and P.K. Townsend, Phys. Rev. D63 (2001) 085001, hep-th/0007124.

[15] J.P. Gauntlett, D. Tong and P.K. Townsend, Phys. Rev. D64 (2001) 025010, hep-th/0012178.

[16] D. Tong, Phys. Rev. D66 (2002) 025013, hep-th/0202012.

[17] K.S.M. Lee, Phys. Rev. D67 (2003) 045009, hep-th/0211058.

[18] M. Arai, M. Naganuma, M. Nitta and N. Sakai, Nucl. Phys. B652 (2003) 35, hep-th/0211103; "BPS Wall in N=2 SUSY Nonlinear Sigma Model with Eguchi-Hanson Manifold" in Garden of Quanta - In honor of Hiroshi Ezawa, Eds. by J. Arafune et al. (World Scientific Publishing Co. Pte. Ltd. Singapore, 2003) pp299-325, hep-th/0302028.

[19] M. Arai, M. Nitta and N. Sakai, "Vacua of Massive Hyper-Kähler Sigma Models of NonAbelian Quotient", hep-th/0307274; "BPS Domain Walls in Massive Hyper-Kahler Sigma Models", to be published in the Proceedings of the 3rd International Symposium on Quantum Theory and Symmetries (QTS3), hep-th/0401084; "Massive Hyper-Kahler Sigma Models and BPS Domain Walls", to be published in the Proceedings of the International Conference on Symmetry Methods in Physics (SYM-PHYS10), hep-th/0401102.

[20] E. Abraham and P.K. Townsend, Phys. Lett. B291 (1992) 85.

[21] J.P. Gauntlett, R. Portugues, D. Tong and P.K. Townsend, Phys. Rev. D63 (2001) 085002, hep-th/0008221.

[22] M. Naganuma, M. Nitta and N. Sakai, Grav. Cosmol. 8 (2002) 129, hep-th/0108133. 
[23] R. Portugues and P.K. Townsend, JHEP 0204 (2002) 039, hep-th/0203181.

[24] M. Naganuma, M. Nitta and N. Sakai, Phys. Rev. D65 (2002) 045016, hep-th/0108179.

[25] Y. Sakamura, Nucl. Phys. B656 (2003) 132, hep-th/0207159; JHEP 0304 (2003) 008, hep-th/0302196.

[26] A. Galperin, E. Ivanov, S. Kalitzin, V. Ogievetsky and E. Sokatchev, Class. Quantum Grav. 1 (1984) 469; A. Galperin, E. Ivanov, V. Ogievetsky and E. Sokatchev, Class. Quantum Grav. 2 (1985) 601; Class. Quantum Grav. 2 (1985) 617.

[27] A. Galperin, E. Ivanov, V. Ogievetsky and E. Sokatchev, Comm. Math. Phys. 103 (1986) 515.

[28] A. Galperin, E. Ivanov, V. Ogievetsky and P.K. Townsend, Class. Quantum Grav. 3 (1986) 625.

[29] A. Galperin, E. Ivanov, V. Ogievetsky and E. Sokatchev, "Harmonic Superspace", Cambridge University Press, Cambridge (2001), 306p.

[30] V.P. Akulov, D.P. Sorokin and I.A. Bandos, Mod. Phys. Lett. A3 (1988) 1633.

[31] A. Taub, Ann. Math. 53 (1951) 472; E. Newman, L. Tamburino and T. Unti, J. Math. Phys. 4 (1963) 915; S.W. Hawking, Phys. Lett. A60 (1977) 81.

[32] T. Eguchi and A.J. Hanson, Phys. Lett. B74 (1978) 249; Ann. Phys. 120 (1979) 82.

[33] E. Ivanov, S. Ketov and B. Zupnik, Nucl. Phys. B509 (1998) 53, hep-th/9706078.

[34] S. Ketov and C. Unkmeir, Phys. Lett. 422B (1998) 179, hep-th/9710185.

[35] G.W. Gibbons, D. Olivier, P.I. Ruback and G. Valent, Nucl. Phys. B296 (1988) 679.

[36] B. Zumino, Phys. Lett. B87 (1979) 203.

[37] J. Wess and J. Bagger, "Supersymmetry and Supergravity", 2nd eds., Princeton University Press (1992), 259p. 
[38] A. Galperin, E. Ivanov, V. Ogievetsky and E. Sokatchev, Ann. Phys. 185 (1988) 1; Ann. Phys. 185 (1988) 22.

[39] N. Ohta, H. Sugata and H. Yamaguchi, Ann. Phys. 172 (1986) 26.

[40] G.W. Gibbons and P.J. Ruback, Comm. Math. Phys. 115 (1988) 267.

[41] G.W. Gibbons and S.W. Hawking, Phys. Lett. B78 (1978) 430.

[42] A. Galperin, E. Ivanov and V. Ogievetsky, Phys. Scr. T15 (1987) 176.

[43] G. Papadopoulos and P.K. Townsend, Nucl. Phys. B444 (1995) 245, hep-th/9501069.

[44] E. Ivanov and G. Valent, Nucl. Phys. B576 (2000) 543, hep-th/0001165.

[45] P.-Y. Casteill, E. Ivanov and G. Valent, Nucl. Phys. B627 (2002) 403, hep-th/0110280.

[46] J. Bagger and E. Witten, Nucl. Phys. B222 (1983) 1.

[47] A. Galperin, E. Ivanov, V. Ogievetsky and E. Sokatchev, Class. Quantum Grav. 4 (1987) 1255.

[48] J. Bagger, A.S. Galperin, E.A. Ivanov and V.I. Ogievetsky, Nucl. Phys. B303 (1988) 522. 